\title{
Molecular Field Theory of Hysteresis in Helix-Coil Transitions of Polynucleotides
}

\author{
G. WEISBUCH* and E. NEUMANN, $\uparrow$ \\ Polymer Department, Weitzmann Institute, Rehovot, Israel
}

\section{Synopsis}

A molecular field theory, taking into account long-range electrostatic forces is used to study helix-coil transitions of polynucleotides. The theory predicts the existence of hysteresis when the electrostatic interaction parameter is large compared to the thermal energy. The theory is applied to the acid-base titration of poly $(\mathrm{A}) \cdot 2 \mathrm{poly}(\mathrm{U})$.

\section{INTRODUCTION}

The influence of cooperativity on conformational changes in biopolymers has been extensively studied both experimentally and theoretically. ${ }^{1}$ Nevertheless relatively small attention has been paid to the existence of hysteresis cycles in some of these transitions. This might be due to the fact that non-reversible transitions are rather suspicious to many scientists. It is the merit of Aharon Katchalsky to have recognized the importance of hysteresis phenomena, especially as a mechanism for molecular memory recording. ${ }^{2}$

The aim of this paper is to present a theory of hysteresis in polyelectrolytes and to apply it to the helix-coil transition of the three-stranded complex poly (A) $\cdot 2$ poly $(\mathrm{U})$ into the constituent polymers polyriboadenylate (poly(A)), and polyribouridylate (poly(U)). This conformational change preceeds the formation of the helix $\operatorname{poly}(\mathrm{A}) \cdot \operatorname{poly}(\mathrm{A})$ in the acid titration of $\operatorname{poly}(\mathrm{A}) \cdot 2$ poly(U) according to Eq. (1):

$$
\operatorname{poly}(\mathrm{A}) \cdot 2 \operatorname{poly}(\mathrm{U}) \stackrel{\mathrm{H}^{+}}{\rightleftarrows} \frac{1}{2} \operatorname{poly}(\mathrm{A}) \cdot \operatorname{poly}(\mathrm{A})+2 \operatorname{poly}(\mathrm{U})
$$

The pronounced hysteresis loop observed in this system ${ }^{3}$ is represented in Figure 1, where potentiometric and spectrophotometric titration data are expressed in terms of the fraction of dissociated (A.2U) segments. In a multistranded helix like poly $(A) \cdot 2$ poly $(U)$ the nucleotide segments can

* On leave of absence from Departement de Physique, U.E.R. de Luminy, 70 route Léon-Lachamp, 13288 Marseille Cedex 2.

† On leave of absence from Max-Planck-Institut für biophysikalische Chemie, 3400 Göttingen Am FaBberg, Germany.

(C) 1973 by John Wiley \& Sons, Inc. 


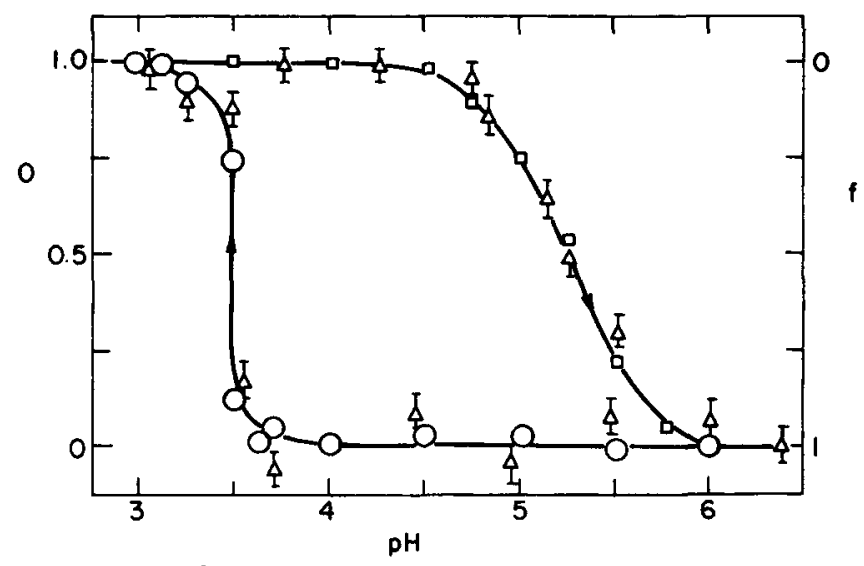

Fig. 1. Experimental determination of $O$, the fraction of open bases in poly(A).2 poly $(\mathrm{U})$ as a function of $\mathrm{pH}^{3}{ }^{3} \mathrm{O}$ and $\square$ potentiometric titration, $\varangle$ spectrophotometric titration. Temperature is $20^{\circ} \mathrm{C}$, concentration of $\mathrm{NaCl} 0.1 M$. The lower branch of the cycle corresponds to the decrease of the $\mathrm{pH}$, the upper branch to the increase of the $\mathrm{pH}$.

be in two different states, either closed or open. In the closed state an adenine base is associated with two uracils by H-bonds (see Fig. 2). This specific association is a condition for maximum stacking interactions in the helix. In the open state, $\mathrm{H}$-bonding between opposite bases is interrupted and stacking interactions are mostly reduced.

The free energy difference between the closed and the open state of a base can be divided into two terms: (i) a stabilizing term due to $\mathrm{H}$-bonding and base stacking, and (ii) a destabilizing term resulting from electrostatic repulsion between the charged phosphate groups of opposing strands.

These two terms do not vary in the same way upon partial opening of the triple helix. The stabilization energy of a nucleotide is completely lost when this nucleotide opens. The electrostatic repulsion term, however, decreases only when the nucleotides are able to move apart from each other. This means that the second term is strongly dependent upon

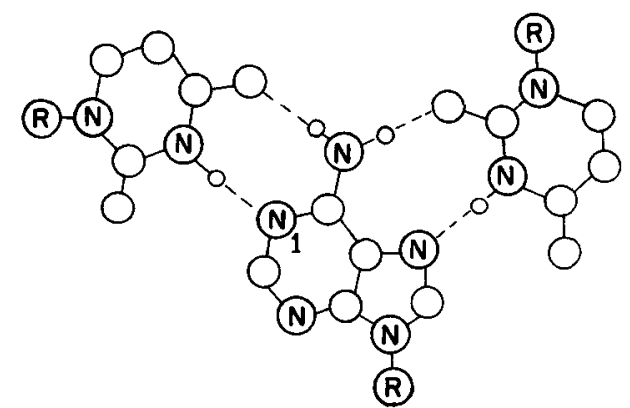

Fig. 2. Schematic representation of the (U.A.U.) base pairing in a poly(A) 2 poly(U) segment. At acidic $\mathrm{pH}$ the $\mathrm{N}_{1}$ nitrogen is protonated, and is no longer available for hydrogen bonding. The adenine thus remains open. 


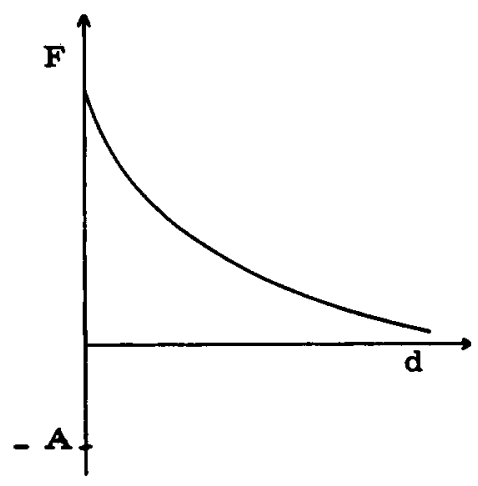

Fig. 3. Free energy $F$ of a nucleotide in a polynucleotide helix as a function of the distance $d$ of the charged phosphate group form the axis of the helix. In the case represented, the free energy of the closed nucleotide is less than the free energy of an open nucleotide far from the axis. The multistranded helix would then be the stable state and the single strands the metastable state.

the state of the neighboring nucleotides. When many consecutive nucleotides are open, they are able to move apart from the triple helix axis and their electrostatic energy is appreciably decreased. However an isolated open nucleotide has nearly the same electrostatic energy as when it is closed. Since it has lost its free energy of stabilization, it has then a relatively high energy. This dependence of the electrostatic energy of an open nucleotide upon the state of its neighbors is the origin of the energy barrier which can create metastability of the closed state relative to the open state of the system. Since the opening of the first nucleotide of a loop is energetically unfavorable, there exists a nucleation barrier similar to the nucleation barrier, due to surface tension, which is responsible for supercooling or superheating in liquid-gas, or liquid-solid, transitions (see Figs. 3 and 4).

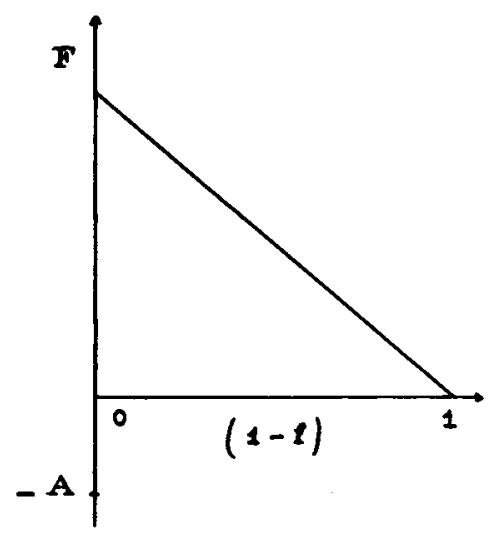

Fig. 4. Average free energy $F$ of an open segment as a function of the fraction of closed segments according to the linear molecular field approximation. 
In conclusion, interaction between neighboring nucleotides involves more than the nearest neighbors for two reasons: (1) The electrostatic forces, even screened, are rather long range forces: at a concentration of $0.1 M$ $\mathrm{NaCl}$, in the case of poly $(\mathrm{A}) \cdot 2$ poly $(\mathrm{U})$, thirty bases are within the range of one Debye radius from a given phosphate group. (2) The electrostatic energy decreases appreciably in the opening process only if several neighboring nucleotides are already open. Nearest-neighbor models are thus inadequate to describe the phenomenon since one has to take into account long-range interactions.

Taking the opposite approach we propose to neglect short-range correlations and to take into account only long-range interactions. We shall consider that an open base is submitted to an electrostatic field depending only upon the degree of opening of the overall complex. This approach is called in physics a molecular field approach from the Weiss theory of ferromagnetism. ${ }^{4}$ It has been already used, on a purely phenomenological basis, to study the cooperativity of biological membranes by Changeux et $a .^{5}$

We shall develop the method in the next section. Apart from its simplicity, one of its main advantages over the usual calculations of partition functions is that it can explain the occurrence of hysteresis.

\section{THEORETICAL MODEL}

We shall treat the case of a double helix and shall suppose that a nucleotide can exist in two different states, closed state or open state.

Closed state. The free energy of the closed state is $-A$.

Open state. The free energy of a nucleotide in the open state far apart from the other strand is taken as the reference free energy. We know that the free energy of a nucleotide depends upon its distance from the other strand, and that it is maximum when this distance is minimum. We shall then consider a mean free energy of the open nucleotides depending upon their mean distance from the other strand, which is supposed to depend only upon the mean opening of the double helix $(1-f)(f$ is the fraction of closed nucleotides). We shall furthermore suppose that the dependence of the energy upon the degree of opening is linear. This is the simplest possible dependence and we have no argument to prove its validity when $f$ varies between 1 and 0 . But we can at least take it as a linear approximation when $f$ is not much different from 1 . For several other reasons we shall see that our calculations are valid mainly when $f$ is close to 1 . Taking $B$ as the proportionality constant we write that the free energy of the open nucleotides

$$
F_{\text {open nucleotides }}=B f
$$

In this respect our approximation can be compared to the concept of molecular field introduced by Weiss. ${ }^{4}$ Weiss writes that the influence of the surrounding magnetic moments on an elementary magnet can be de- 
scribed as an internal molecular field proportional to the mean magnetic moment per unit volume. He then writes that the energy of an elementary magnet is

$$
E=-\boldsymbol{u}_{\mathrm{B}} \cdot \mathbf{M}
$$

where $\boldsymbol{\mu}_{B}$ is the elementary magnetic moment and $\mathbf{M}$ the intensity of magnetization, i.e., the magnetic moment per unit volume.

The free energy $F$ of the system is the sum of the contributions from the two states, plus a lowering term due to the mixing entropy of the two states:

$$
F=-A f+B f(1-f)+k T[f \ln f+(1-f) \ln (1-f)]
$$

$f$ is obtained by differentiating $F$ with respect to $f$. At equilibrium

$$
\frac{d F}{d f}=-A+B(1-2 f)+k T \ln \frac{f}{1-f}=0
$$

and

$$
f=\left[1+\exp \left(\frac{-A+B(1-2 f)}{k T}\right)\right]^{-1}
$$

which we write

$$
f=\frac{1}{1+\exp (-\beta f+\alpha)}
$$

where

$$
\alpha=\frac{-A+B}{k T}
$$

and

$$
\beta=\frac{2 B}{k T}
$$

The equation can be solved graphically by taking the intersection of the first bissectrix $y=f$ with the curve $y=\phi(f)=[1+\exp (-\beta f+\alpha)]^{-1}$ which is very similar to a Fermi-Dirac distribution function ${ }^{6}$ (see Fig. 5). Depending on the respective values of $\alpha$ and $\beta$ there can be either one, two, or three solutions. We have, in fact, more than one solution for certain values of $\alpha$, only if the maximum slope of the Fermi Dirac function is greater than 1 . Since the slope is $\beta / 4$ at the inflection point, we see that a necessary condition for the existence of three solutions is $\beta>4$ which means $B>2 k T$.

Let us describe the different situations occurring when $\alpha$ is increased from a large negative value to a large positive value, $\beta$ being constant and larger than 4. (This is realized experimentally by varying the $\mathrm{pH}$ of the solution.) Figure 5 represents the intersection of the two curves for different values of $\alpha$ and for $\beta=5$. 


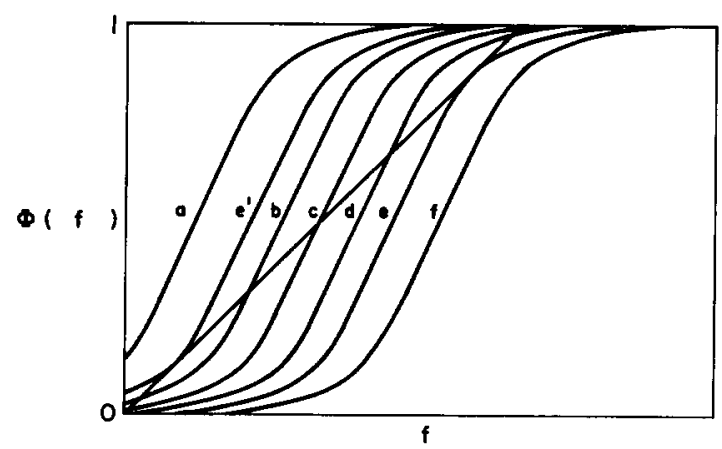

Fig. 5. Graphical solution of the equation $f=\phi(f)=(1+\exp (-\alpha+\beta f))^{-1}$ in the case $\beta=5$. The curve $\phi(f)$ correspond to different values of the parameter $\alpha$ : For curve $a, \alpha=1 ; e^{\prime}, \alpha=1.6 ; b, \alpha=2 ; c, \alpha=2.5 ; d, \alpha=3 ; f, \alpha=4$.

(a) When $\alpha<0$ the straight line cuts the Fermi Dirac function only in one point where $f \simeq 1$. The helix is nearly completely closed.

(b) When $\alpha$ increases one reaches situations like (b) where the straight line cuts the curve in the three points. One can understand the meaning of these points by coming back to the free energy $F$ as a function of $f$ (Fig. 6). The three points of intersection correspond to the three extrema of $F$ : the two extrema values of $f$ correspond to minima of $F$ and the inter-

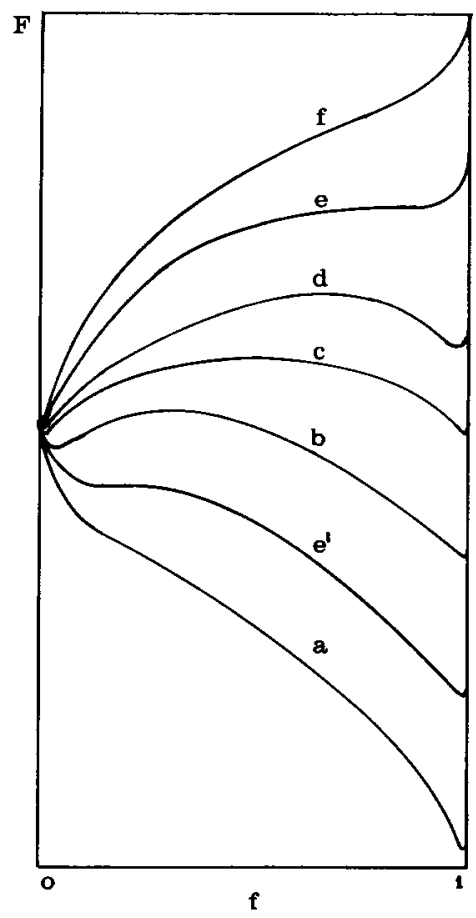

Fig. 6. The free energy $F$ as a function of $f$ for given $\beta$ and $\alpha$. The notations of the curves $a, b \ldots f$ correspond to those of Fig. 5 . 
mediate value of $f$ to a maximum. The extreme values correspond then to stable or metastable state while the intermediate value is unstable. The metastable state is the state with the relatively higher free energy. Since the Fermi Dirac function is symmetric about the point $f=1 / 2$ one can deduce that the stable state is the nearly closed state when $\alpha<\beta / 2$ and the nearly open state when $\alpha>\beta / 2$. However if one starts from the closed state, as long as the energy barrier exists, the system remains in the nearly closed state when $\alpha$ increases. The helix opens up only when the barrier disappears, which occurs when the straight line is tangent to the curve (situation e). (f) In situation $\mathrm{f}$, there is again only one solution, the almost open solution.

If one now starts from the open state (f), and decreases $\alpha$, the same barrier that prevented a transition from closed to open state will now prevent a transition from open to closed state until point $e^{\prime}$, different from $e$, where the helix transits to the nearly closed state.

The coordinates of the points $e$ and $e^{\prime}$ are obtained by solving the equation

$$
\phi^{\prime}(f)=\frac{\beta \exp (\alpha-\beta f)}{[1+\exp (\alpha-\beta f)]^{2}}=1
$$

by substituting

$$
z=\exp (\alpha-\beta f)
$$

this equation becomes

$$
z^{2}+(2-\beta) z+1=0
$$

The two solutions are:

$$
z=\frac{\beta}{2}-1 \pm \sqrt{\frac{\beta^{2}}{4}-\beta}
$$

Approximate solutions in the limit $\beta \gg 1$ are: for point $e$

$$
\begin{aligned}
z & =\frac{1}{\beta-2}, f=1-\frac{1}{\beta} \\
\alpha & =\beta-1-\ln (\beta-2)
\end{aligned}
$$

for point $e^{\prime}$

$$
\begin{gathered}
z=\beta-2, f=\frac{1}{\beta-1} \\
\alpha=\frac{\beta}{\beta-1}+\ln (\beta-2)
\end{gathered}
$$

We are now able to sketch the hysteresis cycle showing the variation of $f$ with $\alpha$ (Fig. 7). Upon increasing $\alpha$, opening occurs at point $e$ (lower 


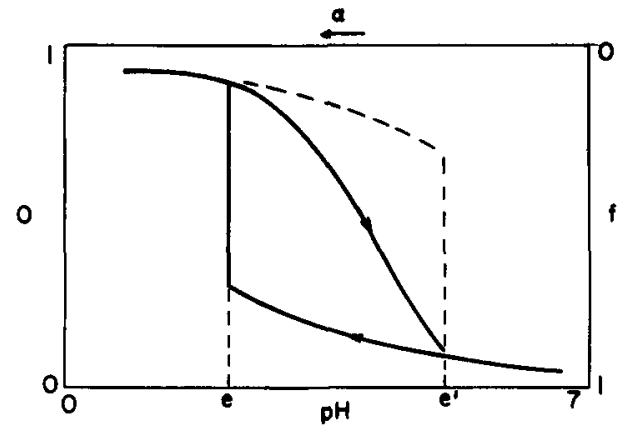

Fig. 7. The hysteresis cycle as predicted by theory. $O$ is the fraction of open bases and $f$ the fraction of closed bases. The dotted line is what one would expect, not taking into account the change of $\beta$ when protonation occurs.

curve) whereas upon decreasing $\alpha$, closing occurs at point $e^{\prime}$ (upper dotted curve).

In conclusion, our model predicts the existence of sharp transitions and hysteresis when $\beta>4$. There exists then a critical temperature $T_{\mathrm{c}}=$ $B / 2 k$ above which hysteresis is not observed any more.

\section{APPLICATION OF THE MODEL TO POLY A.2 POLY U \\ Opening of the Triple Helix}

The opening of poly $A \cdot 2$ poly $U$ can be described by the following steps

$$
\mathrm{A} \cdot 2 \mathrm{U} \rightleftarrows \mathrm{A}+2 \mathrm{U} \quad \text { opening reaction }
$$

$\mathrm{A}+\mathrm{H}^{+} \rightleftarrows \mathrm{AH}^{+} \quad$ protonating reaction

$$
2 \mathrm{AH}^{+} \rightleftarrows\left(\mathrm{AH}^{+}\right)_{2} \quad \text { formation of the double helix }
$$

Each of these partial reactions refers to bases and not to the entire polymer.

In the process of opening, the number of open bases remains small until the occurrence of the vertical transition. The formation of the double helix involves two bases of two different triple helices which tend to repel each other electrostatically. For these two reasons we shall neglect the formation of the double helix as long as the triple helix has not changed to the almost open state.

We remain then with the opening reaction which is shifted towards the righthand side by protonation. We can approximate the extent of protonation by using an equilibrium constant $C$

$$
\frac{[\mathrm{A}]\left[\mathrm{H}^{+}\right]}{[\mathrm{AH}+]}=C
$$

This relation is only an approximation for a polyacid because the occurrence of protonation on a site changes the electrostatic energy on the neighboring sites thus changing $C$. But since we restrict ourselves to the beginning of 
the opening process we shall take $C$ as a constant until the transition point $e$. Thus the proportion of protonated to non-protonated open bases is

$$
\frac{\left[\mathrm{AH}^{+}\right]}{[\mathrm{A}]}=\frac{\left[\mathrm{H}^{+}\right]}{[\mathrm{C}]}
$$

From Eq. (1) we know that the fraction of closed bases to open nonprotonated is

$$
\frac{[A \text { closed }]}{[A]}=\exp \frac{A-B(1-2 \mathrm{f})}{k T}
$$

then

$$
\begin{aligned}
f=\frac{[\text { A closed }]}{[\mathrm{A} \text { closed }]+[\mathrm{A}]+\left[\mathrm{AH}^{+}\right]} & {[\mathrm{A}] \exp \frac{A-B(1-2 f)}{k T} } \\
= & \frac{\mathrm{A}-\mathrm{B}(1-2 \mathrm{f})}{k T}+[\mathrm{A}]+\frac{\left[\mathrm{H}^{+}\right]}{C}[\mathrm{~A}]
\end{aligned}
$$

and

$$
f=\left[1+\left(1+\frac{\left[\mathrm{H}^{+}\right]}{C}\right) \exp \frac{-A+B(1-2 f)}{k T}\right]^{-1}
$$

To compare with the theoretical model we take $\alpha$ as

$$
\alpha=\frac{-A+B}{k T}+\ln \left(1+\frac{\left[\mathrm{H}^{+}\right]}{C}\right)
$$

and

$$
\beta=2 B / k T
$$

This shows how $\alpha$ can be driven by the $\mathrm{pH}$, a decrease of $\mathrm{pH}$ giving rise to an increase of $\alpha$.

How well does an experimental curve like the one on Figure 1 fit with the equations? Is it possible for instance to predict the coordinates of the point of transitions $e$ ? This needs the knowledge of the three constants $A, B$, and $C$ appearing in the equations.

$A$ can be calculated from the heat and the temperature of transition of poly (A) $\cdot 2$ poly(U). In the conditions of Figure 1 (temperature $20^{\circ} \mathrm{C}$, ionic salt concentration $0.1 N$ ) we obtain $A$ by linear interpolation of the data of Krakauer and Sturtevant. ${ }^{7}$ The linear interpolation consists in supposing that the entropy of fusion is constant over an interval of $30^{\circ} \mathrm{C}$ and that $A$ depends linearly on the logarithm of the concentration of ionic salt (Kotin's approximation). ${ }^{8}$

$$
A\left(0.1,20^{\circ} \mathrm{C}\right)=1 \mathrm{kcal} / \mathrm{mole}
$$


$B$ has not been calculated directly. A rough estimate of its order of magnitude is given in the appendix. But we can determine it from the value of $f$ at the transition point (Fig. 1) $0.12<1-f<0.2$. (This large imprecision is due to two reasons: polydispersity of the sample, and the fact that since acid drops are added into the solution, the local $\mathrm{pH}$ is in some places less than the $\mathrm{pH}$ of transition. Since the closed state is metastable this give rise to some irreversible transitions before the $\mathrm{pH}$ of the solution reaches the $\mathrm{pH}$ of transition.)

This value of $f$ at the transition gives $5<\beta<8$. If we take $\beta=6$ we obtain $B=1.8 \mathrm{kcal}$ per mole.

As explained above, $C$ is difficult to determine. If we take $\mathrm{pK}=\mathrm{colog}$ $C=4.5$. (The pK generally admitted for poly A but never actually directly measured $)^{9}$ we find the $\mathrm{pH}$ of transition by solving the equation.

$$
\begin{aligned}
\alpha & =\beta-1-\ln \beta-2 \\
\frac{-A+B}{k T}+2.3(\mathrm{pK}-\mathrm{pH}) & =\beta-1-\ln (\beta-2) \\
\mathrm{pH} & =3.5
\end{aligned}
$$

This is the same value as found experimentally. Thus the proposed model predicts the lower branch of the hysteresis curve fairly well.

\section{Closing of the Triple Helix}

In describing the opening of the triple helix we have neglected the influence of protonation on $\beta$. In fact, protonation, by decreasing the charge of the strand, decreases $\beta$. Since the degree of opening before the transition is small, the assumption that $\beta$ is constant is justified. The situation is completely different when one starts from the open state. The double helix poly $\mathbf{A}$.poly $\mathbf{A}$ is almost electrically neutral since the charge of the proton compensates the charge of the phosphate group. This means that the electrostatic barrier does not exist at the beginning of the closing process of the triple helix and that it is fully reestablished only after deprotonation has been performed. Since hysteresis exists only when $\beta>4$ the closing branch of the hysteresis curve looks much more like an equilibrium curve and does not present any sharp transition. Instead of the dotted line which would correspond to the constant $\beta$ model we rather expect the continuous line (Fig. 5) corresponding to the gradual increase of $\beta$ with $\mathrm{pH}$. We are then able to understand at least the qualitative behavior of the upper branch in Figure 1.

\section{CONCLUSIONS}

Let us first recall that our model is based on several simplifying assumptions: (1) We have taken into account only long-range interactions, thus neglecting short range correlations. (2) We have used a linear approximation of the molecular field. (B) All the numerical applications as well 
as some formulas rest in the $\beta \gg 1$ approximation. When $\beta$ is close to 4 we are approaching the critical region. It is a well-known fact that molecular field theories are unable to give a quantitative description of critical phenomena. ${ }^{4}$

Within these assumptions the molecular field theory is sufficient to describe the hysteresis cycle of $\operatorname{poly}(\mathrm{A}) \cdot 2 \operatorname{poly}(\mathrm{U})$. Furthermore from the measured value of $\beta$, we are able to explain the absence of hysteresis for the poly $(\mathrm{A}) \cdot \operatorname{poly}(\mathrm{U})$ double-helix titration: the repulsive field experienced by a strand in a double helix is roughly half the field experienced by $\operatorname{poly}(\mathrm{A})$ in $\operatorname{poly}(\mathrm{A}) \cdot 2$ poly $(\mathrm{U})$.

Then we expect that

$$
\beta_{\text {double helix }} \simeq \frac{\beta(\operatorname{poly}(\mathrm{A}), \operatorname{poly}(2 \mathrm{U}))}{2} \simeq 3
$$

Since $\beta_{\mathrm{A} \cdot \mathrm{U}}<4$, no hysteresis is expected.

In conclusion, the molecular field approach appears to be a powerful tool to study cooperativity in polymers with predominantly long-range forces, particularly polyelectrolyte systems. The molecular field theory is currently being used as a basis for the analysis of hysteresis phenomena observed in lipoprotein membranes and globular proteins with a high density of surface charges.

\section{APPENDIX}

\section{Evaluation of B}

To evaluate $B$, we have to calculate the electrostatic energies of the different configurations corresponding to the same $f$ and then to take the mean energy by averaging each energy with the probability of occurrence of the corresponding configurations.

$$
E_{(\jmath)}=\sum_{i} E_{i} p_{i}
$$

$i$ corresponds to configurations having the same energy. Let us examine the energies corresponding to loops of $1,2,3, \ldots n$ open consecutive segments.

If $n$ is large we shall admit that the phosphate charges lie on a circle in order to increase their mutual distance and to minimize their electrostatic energy. If $n$ is small the circle will be elongated due to the stronger repulsion by the double or triple charged helix.

If we still retain the circle approximation the radius of a loop of $n$ segments is approximately

$$
r=\frac{(n+1) a}{\pi}
$$

where $a$ is the interphosphate distance. The mean deviation due to opening is then

$$
d=\frac{1}{\pi} \int_{0}^{\pi} \sin \theta r d \theta=\frac{2 r}{\pi}=\frac{2}{\pi^{2}}(n+1) a
$$

The mean energy of a segment in a loop of $n$ open segments is then

$$
E_{n}=U-\frac{2}{\pi^{2}} \frac{(n+1)^{2}}{n} \epsilon a
$$


where $U$ is the electrostatic energy of a closed segment and $\epsilon$ is the electric field at the surface of the helix (the factor $(n+1) / n$ comes from the fact that a loop of $n$ open segments contains $(n+1)$ phosphates).

When one neglects the short-range correlations, the probability that an open nucleotide belongs to a loop of one open nucleotide becomes:

$$
P_{1}=f^{2}
$$

(It is the probability for its two neighbors to be closed.)

The probability that an open nucleotide belongs to a loop of $n$ open nucleotide is

$$
P_{n}=f^{2} n(1-f)^{n-1}
$$

(It is the probability of having $n$ consecutive neighbors open, $(1-f)^{n-1}$, multiplied by $n$, because the given base can be in $n$ positions in this loop, and multiplied by the probability that the two nucleotides at the ends of the loop are closed.)

Then

$$
\begin{aligned}
\langle E\rangle & =\sum_{1}^{+\infty} P_{n} E_{n}=U-\frac{2 \epsilon a}{\pi^{2}} f^{2} \sum_{1}^{+\infty}(n+1)^{2}(1-f)^{n-1} \\
& =U-\frac{2 \epsilon a}{\pi^{2}}\left(f+1+\frac{2}{f}\right)
\end{aligned}
$$

When $(E)$ is developed as a series in $0=(1-f)$ with $f$ close to one we have

$$
\langle E\rangle=U-\frac{2 \epsilon a}{\pi^{2}}(4+0)
$$

which gives $B=2 \epsilon a / \pi^{2}$.

This calculation is rather rough but it shows that the molecular field theory, neglecting short-range interactions, predicts a linear dependence of the mean energy of an open base as a function of $f$, when $f$ is close to 1 . The linear dependence is only an approximation limited to the small deviation from 1 . We can try to calculate the order of magnitude of $B$.

Let us first remark that we have overestimated the deviation of the isolated open base (see above remark). If we take it as $0, \mathrm{~B}$ becomes $9_{\epsilon a} / \pi^{2}$. We can then take as an intermediate value $B=\epsilon a / 2$ per base and $B=3 \epsilon a / 2$ per segment for the triple helix. The electric field at the surface of the triple helix is $\lambda / 2 \pi d \epsilon$ where $\lambda$ is the charge per unit length, $d$ the helix diameter, and $\epsilon$ the dielectric constant.

If we neglected screening at the surface of the polymer, we obtain

$$
B \simeq 0.25 \mathrm{eV}
$$

If we take into account screening, following calculation by M. T. Record, ${ }^{10}$ this result has to be divided by 7 giving then $B \simeq 0.034 \mathrm{eV}$.

The calculations of Record are based on linearization of the Poisson-Boltzmann equation, which is not valid near the helix where the electrostatic energy is higher than $k T$. Since these calculations predict a value of the field which is to low, it seems reasonable to give for $B$ an order of magnitude of $50-100 \mathrm{mV}$ to be compared with $2 k T=$ $50 \mathrm{meV}$.

We acknowledge the proposal of this study of hysteresis in polynueleotides by the late Aaron Katchalsky. We thank Dr. A. Revzin and Mrs. M. Spodheim for helpful discussions. One of us (G.W.) thanks the Centre National de la Recherche Scientifique and the European Molecular Biology Organisation for financial support. 


\section{References}

1. Poland and Scheraga, Theory of Helix-Coil Transitions in Biopolymers, Academic Press, New York, 1970.

2. A. Katchalsky and E. Neumann, Intl. J. Neuroscience, 3, 175 (1972).

3. E. Neumann and A. Katchalsky, Ber. Bunsenges physik Chem., 74, 868 (1970).

4. See for instance R. Brout, Phase Transitions, W. A. Benjamin, New York, (1965).

5. J. P. Changeux, J. Thiery, Y. Tung, and C. Kittel, Proc. Natl. Acad. Sci., 57, 335 (1967).

6. See for instance C. Kittel, Thermal Physics, Wiley, New York (1969).

7. H. Krakauer and J. Sturtevant, Biopolymers, 6, 486 (1968).

8. L. Kotin, J. Mol. Biol., 7, 309 (1963).

9. R. A. Cox, Biochem. J., 100, 146 (1966).

10. M. T. Record, Biopolymers, 5, 975 (1967).

Received November 1972

Revised March 6, 1973 